Wales, which it has been our duty to publish in fiemf's Mrran. tile Gazette during the past eleven year: :-

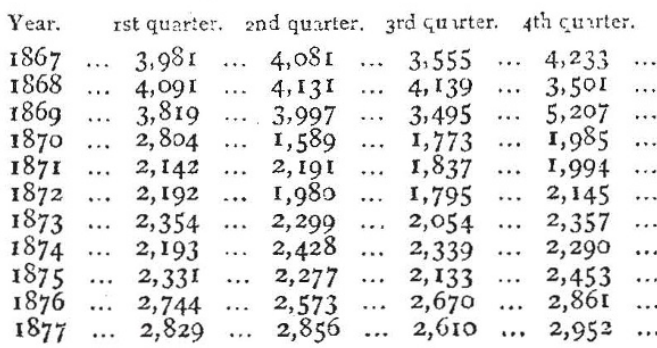

Totals. I 5,850 I 5,862 I 6,518 $8,15 I$ 8,164 8,112 9,064 9,250 9,194 9,194
I0,848 I 1,247

Total for II years .. 122,260

"The question occurs: Does the number of failures in a year de pend upon natural causes? that is to say, Would the number rise and fall periodically according to the state of trade (or national prosperity) if the Bankruptcy Law remained constant? Whenever failures have become frequent, complaints have been made against the Law, and not without reason, but many who com. plain ignore the existence of any other cause. We compared the foregoing figures with the scientific tables recently published in NATURE, from the pen of Prof. Balfour Stewart, ${ }^{1}$ and, being struck with the coincidence in their fluctuations, we further compared them with the statistics publislued by Messrs. Dun and Co., of New York, of the failures in the United States during the past eight years, ${ }^{2}$ which period, being that of the existence of our present Bankruptcy Law, affords us a fair opportunity for making a comparison. Messrs. Dun and Co. report the following as the total failures in the United States during this period :-

$\begin{array}{llcc}1870, \text { Number of } & \text { Failures, } 3,551 \\ 1871, & , & , & 2,915 \\ 1872, & , & , & 4,069 \\ 1873, & ", & , & 5,183 \\ 1874, & , & , & 5,830 \\ 1875, & , & , & 7,740 \\ 1876, & , & , & 9,092 \\ 1877, & , & , & 8,822\end{array}$

"Evidentiy the same causes which were at work in England to depress trade and overwhelm the struggling and improvident classes, were equally effective in other countries-similar complaints of depression come to us from every part of the globe.

" The discussion which has arisen out of Dr. Hunter's suggestion of a 'famine period' in India, has brought to the public some knowledge of the existence of natural periods or cycles, of an average duration of $I I^{\circ} 9$ years each. The suggestion that England is affected with the same regularity is but reasonable, and although fortunately for us as a nation the effects do not produce famine, it appears evident that some degree of suffering is caused, and that the number of failures is thereby materially increased-the commercial panics which have occurred with about the same regularity furnish further evidence that this is the case.

"If we make due allowance for the excessive number in the last quarter of 1869 , caused by the change in the Law, we find that the maximum number of failures in the last cycle occurred in the year 1868 , which was the year succeeding the natural minimum; hence we may conclude that about a year is required for the full effect of the natural depression to be reproduced in comamerce. The twelve months from October 1,1867 , to September 30,1868 , appear to have been more serious to commercial men than either of the complete years, according to the number of failures :-

$$
\begin{aligned}
& \text { "In the } 4^{\text {th }} \text { Quarter of } 1867 \text { there were 4,233 failures. }
\end{aligned}
$$

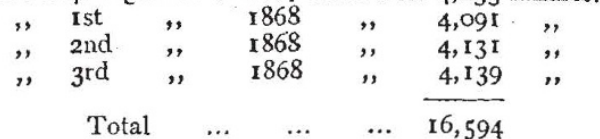

"These data indicate that we have not yet reached the worst of the present period-assuming that it runs an average length,

\footnotetext{
1 Vide NATURE, vol. xyi. pp. $9,26,45$.
2 Messrs. Dun and Co.'s Annual Circular, January, 1878.
}

we have to endure an increasing number of failures which will not reach its maximum until the fourth quarter of $r 879$."

JOHN KEMP AND CO.

Since this was written $I$ have had counted the number of failures gazetted since January 1,1878 , and I find that they are 2,042 in excess of the corresponding period (January 1 to November 19) in the preceding year.

J. K.

\section{Strange Properties of Matter}

The following are two experiments which will, perhaps interest some of your readers :-

Experiment No. 1.-The "Weiding" of Metals at Low Temperatures

Some time ago, "in order to estimate the ifamount of hydrocyanic acid in a solution, I precipitated it with silver nitrate. After having filtered and washed the precipitate, I reduced it to the metallic state by heating to the required temperature. Just as I was about to allow it to cool, I noticed a small piece of dirt among the reduced silver. In order to separate them, I took a thin platinum wire, and pushed the silver to one side, but on attempting to take the wire away the silver remained in contact with it. As I thought this curious, I tried the following experiment. I took a piece of silver foil about one centimetre square, placed it in an inverted porcelain crucible lid, and heated it to about $500^{\circ} \mathrm{C}$.; then I brought into contact with it the extremity of a thin platinum wire, and to my astonishment the wire raised the silver from the lid, and it re mained in contact when cold, as the silver was so very much below its melting-point ; the abore fact caused me some surprise, and I could not satisfactorily account for it.

I wrote to Sir W. Thomson, F.R.S., giving him a description of the above experiment, and in return I received a reply asking me to come and show him the experiment at his laboratory. I accordingly went up to the Glasgow University, and repeated it before him. He was very much interested, and advised me to write to NATURE, giving a description of the experiments. Sir W. Thomson gave the following explanation-That it was a remarkable case of "cohesion," the two metals, in fact, "welding," although the temperature was far below the melting-point of silver. The above experiment can be performed successfully at lower temperatures than $500^{\circ} \mathrm{C}$., if smaller pieces of foil are taken. Other metals, for instance, copper and aluminium, cohere to silver in the same manner as platinum, but less strikingly.

\section{Experiment No. 2,-A Curious Resonator}

Some months ago I made the following experiment:-I took a sma!l tuning-fork and struck it on the table. After the note had died away, so that it was no longer audible, $I$ held the fork in the tip of the flame of a Bunsen burner, when the note was given out, so that it could be heard at some distance. I showed Sir W. Thomson this experiment, who gave the following explanation-That owing to the difference in density of the gases in the flame, the flame acted as a resonator, and so the note was emitted.

It seems to me that experiment No. I could be made the subject of an interesting research, but as $\mathrm{I}$ am wholly engaged in commercial pursuits, I an unable to take it up.

Glasgow, November 12

$$
\text { Charles A. Fawsitt }
$$

\section{Galvanometer for Strong: Currents}

I MUST 'confess that I was surprised by Mr. R. E. Baynes' communication, in NATURE, yol, xix. p. 33, that the galvanometer I have proposed in NATURE, vol. xviii. p. 707, has already been described. Before writing my article I have searched a good many books and journals relating to the subject without finding an allusion to any such instrument. Since Mr. Baynes drew my attention to "The Elements of Physical Manipulation," by Prof. Pickering, of the Massachusetts Institute of Technology, U.S., I have procured this book and find that it certainly does contain the theory of a galvanometer like mine, with the coil moving round a horizontal axis. As far as I know, such an instrument has, however, not been practically employed either in this country or on the Continent before I introduced it, though its want must have been much felt for some time past. 'This seems to show that Prof. Pickering's description of the instrument has not been brought to the general 